\title{
COMPARAÇÃO DE PRODUTOS DE PRECIPITAÇÃO PARA A AMÉRICA DO SUL
}

\section{LUCÍA IRACEMA CHIPPONELLI PINTO ${ }^{1}$, MARCOS HEIL COSTA ${ }^{2}$, FRANCISCA ZENAIDE DE LIMA $^{2}$, LUCIANA MARA FREITAS DINIZ ${ }^{3}$, GILBERTO C. SEDIYAMA ${ }^{2}$ E FERNANDO FALCO PRUSKI $^{2}$}

\author{
${ }^{1}$ Universidade de Buenos Aires - UBA, Departamento de Ciência Atmosféricas e dos Oceanos, \\ Buenos Aires, Argentina \\ ${ }^{2}$ Universidade Federal de Viçosa - UFV, Departamento de Engenharia Agrícola, \\ Viçosa, Minas Gerais, Brasil \\ ${ }^{3}$ Universidade Federal de Minas Gerais - UFMG, Departamento de Ciência da Computação, \\ Belo Horizonte, Minas Gerais, Brasil \\ luchipponelli@hotmail.com
}

Recebido Agosto 2008 - Aceito Junho 2009

\begin{abstract}
RESUMO
Este trabalho compara cinco bancos de dados de precipitação para a América do Sul, para o período 2000 a 2004, considerando a variação meridional, a variação por diferentes bacias hidrográficas (Amazonas, Tocantins, São Francisco, Orinoco, Paraná/Prata, além das bacias dos rios da Patagônia) e pelos principais tipos de vegetação (floresta tropical perene, floresta tropical semi-decídua, caatinga, cerrado e pampas). Foram utilizados três produtos provenientes da composição de dados observados com sensoriamento remoto (TRMM, CMAP e GPCP) e dois de dados de reanálise (NCEP/NCAR e CPTEC). Os resultados indicam que os campos de intensidade média da precipitação anual dos diferentes produtos apresentam comportamento diferenciado entre si. Por exemplo, a reanálise do CPTEC não é capaz de representar os principais regimes de precipitação existentes no continente, demonstrando uma forte tendência em superestimar a intensidade média da precipitação no interior do nordeste brasileiro e subestimá-la na maioria das demais regiões. Os produtos que combinam dados de estações pluviométricas com os de sensoriamento remoto, mostram-se com valores bem próximos do esperado, principalmente o CMAP e GPCP.
\end{abstract}

Palavras-Chave: Precipitação, Reanálise, Série de dados.

ABSTRACT: COMPARISON OF PRECIPITATION PRODUCTS FOR SOUTH AMERICA

This work compares five precipitation datasets for South America, for the period 2000-2004, considering the meridional variation, the variation across the main continental watersheds (Amazonas, Tocantins, São Francisco, Orinoco, Paraná/Prata, in addition to the main Patagônia basins) and across the main vegetation types (tropical evergreen forest, tropical deciduous forest, woodland, savanna and grassland/steppe). We compare three products based on a combination of rain gauge measurements and satellite data (TRMM, CMAP and GPCP) and two based on reanalyzed meteorological datasets (NCEP/NCAR and CPTEC). The results indicate that the annual average precipitation fields of the different products present different behavior among them. For example, the CPTEC reanalysis does not represent the main regimes of precipitation in the continent, overestimating the precipitation in the interior of northeast Brazil and underestimating the precipitation in the rest of the continent. The combined rain gauge-remote sensing products obtained similar patterns, mainly CMAP and GPCP. Keywords: Precipitation, Reanalysis, datasets. 


\section{INTRODUÇÃO}

As pesquisas na área de climatologia, desde as mais simples às mais complexas, necessitam de observações das variáveis meteorológicas, de preferência que representem séries de longos períodos. Mas, mesmo reconhecendo-se a importância deste tipo de observações e apesar dos avanços científicos e tecnológicos pelos quais os sistemas de medições meteorológicas passaram nas últimas décadas, ainda existe, em muitas partes do globo, uma carência considerável de observações meteorológicas que formem séries de longos períodos. Essa lacuna existente nas observações meteorológicas de muitas regiões do planeta devese, em grande parte, à dificuldade em se conseguir observações das variáveis meteorológicas, devido a questões geográficas e econômicas.

Na busca de superar essas dificuldades, pesquisas têm sido feitas visando obter metodologias para gerar longas séries de dados meteorológicos que atendam, de forma satisfatória, a ausência de observações das variáveis meteorológicas a nível global e/ou regional.

No meio científico é possível encontrar, basicamente, três métodos para construir bancos de dados espacializados das variáveis meteorológicas: a interpolação de dados obtidos em estações (New et al., 1999; Legates e Willmott, 1990; Leemans e Cramer, 1990), a técnica de reanálise (Kalnay et al., 1996; Uppala et al., 2005) e a combinação de dados de estações com o sensoriamento remoto (Xie e Arkin, 1997; Huffman et al., 1997; Kummerow et al., 1998). Atualmente, encontram-se disponíveis para a comunidade científica, vários bancos de dados com séries históricas de diversas variáveis geradas por alguma dessas técnicas. Porém, estes bancos de dados podem apresentar discrepâncias quando comparados entre si, principalmente para regiões do planeta onde a confiabilidade dos dados observados é questionável. Costa e Foley (1998) compararam seis bancos de dados de precipitação para a Amazônia, concluindo que, embora as médias de longo prazo para a região fossem semelhantes, os padrões espaciais e a variação temporal eram expressivamente diferentes. Em outra análise, Rao et al. (1996) compararam os dados de precipitação da reanálise do NCEP/NCAR com observações das estações do INMET (Instituto Nacional de Meteorologia) para o nordeste brasileiro, concluindo que as precipitações da reanálise são, geralmente, superiores às observadas nas estações.

Em vista do exposto, este trabalho tem como objetivos: comparar e avaliar a distribuição espacial dos campos de precipitação de diferentes produtos de precipitação para a América do Sul.

\section{DADOS E METODOLOGIA}

Para a comparação dos produtos de precipitação foram utilizados cinco bancos de dados, sendo dois obtidos a partir de reanálise (NCEP/NCAR e CPTEC) e três que combinam dados de sensoriamento remoto com dados de pluviômetros (CMAP, GPCP e TRMM). Este estudo foi realizado para o período de cinco anos (2000 a 2004), período escolhido em função da menor série disponível (reanálise do CPTEC).

\subsection{Dados de Reanálise}

Movidos pela necessidade de solucionar a carência de observações das variáveis meteorológicas, alguns cientistas perceberam que o advento da modelagem numérica poderia se tornar uma ferramenta eficiente para gerar séries históricas das variáveis meteorológicas, com a vantagem de possibilitar sua expansão para regiões mais remotas do globo, onde a observação convencional das variáveis meteorológicas é difícil de ser realizada.

As séries históricas de reanálise, geradas a partir de modelos, são construídas com base em séries de variáveis meteorológicas disponíveis a partir de algum tipo observação. Portanto, as séries de reanálise podem ser originadas a partir da assimilação de dados meteorológicos observados em superfície (estações), aviões, navios, imagens de satélite, imagens de radar e radiossondagens. A técnica de reanálise atmosférica processa, simultaneamente, uma grande variedade de observações, usando as leis físicas contidas nos modelos de previsão do tempo. Sendo assim, o histórico do estado atmosférico pode ser reconstruído dinamicamente.

A quantidade e a qualidade dos dados observados usados para reanálise são cruciais. Por isso, é necessário fazer um controle de qualidade dos mesmos antes da assimilação visando reduzir os erros na reanálise. No início, o índice de acerto apresentado por estes modelos era limitado, o que, geralmente, estava associado ao tratamento inadequado dos processos físicos ocorridos na atmosfera, por exemplo, a formação de nuvens e a quantidade de precipitação que pode haver numa determinada região. Com o passar dos anos observou-se que os modelos apresentam uma sensível dependência em relação à condição inicial (Lorenz, 1969). Por isso, é importante utilizar dados de entrada de boa qualidade no modelo, pois pequenos erros nas condições iniciais podem ocasionar grandes erros nos resultados de saída do modelo. Pohlmann e Greatbatch (2006) mostram que as diferentes reanálises freqüentemente apresentam discordância entre si, principalmente em regiões onde as observações são escassas, como por exemplo, o hemisfério sul, onde há pouca disponibilidade de dados até os dias atuais.

A proposta do projeto de reanálise NCEP/NCAR foi produzir análises globais de campos atmosféricos para atender 
às necessidades das comunidades de pesquisa e monitoramento do clima. Kalnay et al. (1996) descreveram o projeto de reanálise do NCEP/NCAR, que cobre o período de 1948 até a atualidade. As variáveis de saída fornecidas pelo NCEP/NCAR são classificadas em quatro classes, de acordo com o grau de influência do modelo. $\mathrm{O}$ módulo central e a assimilação dos dados usam uma resolução de 28 níveis verticais com uma resolução horizontal de aproximadamente $210 \mathrm{~km}$ (T62). As séries históricas de reanálise do NCEP/NCAR são muito utilizadas em trabalhos que descrevem as condições gerais da atmosfera, seja em análise de áreas isoladas ou para o planeta como um todo. Roads et al. (1999) realizaram um estudo sobre o balanço contínuo de água superficial para os Estados Unidos e concluíram que, para a região estudada, os dados de precipitação da reanálise do NCEP/NCAR era superior àquela obtida através do modelo espectral global do próprio NCEP.

No Brasil, o CPTEC disponibilizou, em 2006, um produto novo de reanálise regional, cobrindo o período de 2000 a 2004, usando o modelo regional Eta. Uma das principais diferenças deste produto em relação a outros centros é o seu sistema de assimilação regional que cobre a América do Sul e oceanos adjacentes, pois geralmente os dados de reanálise são disponibilizados a nível global. Este produto possui uma resolução vertical de 38 níveis e uma resolução horizontal de $40 \mathrm{~km}$. A reanálise regional do CPTEC é um produto recente e ainda pouco explorado em trabalhos científicos. Trata-se de um produto muito promissor, pela sua alta resolução, e por cobrir a América do Sul, onde existe uma escassez de dados observados de boa qualidade. Além disso, é uma região que apresenta uma grande variabilidade de clima com distintas características regionais, podendo-se destacar vários regimes diferentes de precipitação.

\subsection{Dados de Superfície Combinados com Dados de Satélite}

A combinação de dados de superfície com estimativas feitas por satélite é outra opção adotada para criar bancos de dados climatológicos globais. Estes bancos de dados têm se mostrado satisfatórios para analisar a precipitação global, quando comparados com outros produtos, principalmente em regiões com baixa disponibilidade de dados, como os oceanos.

O GPCP (Global Precipitation Climatology Project) é um projeto estabelecido pelo WCRP (World Climate Research Programme) com o objetivo de quantificar a distribuição da precipitação global para uma série longa (Huffman et al., 1997). Este projeto fornece médias mensais de precipitação em uma grade de $2,5^{\circ} \times 2,5^{\circ}$ (lat/lon). Baseia-se no procedimento de análise que mistura várias estimativas para produzir campos de precipitação global. Assim, a análise da grade é baseada em medidas de observações convencionais e satélite para calibrar o banco de dados. Para se gerar estas séries de dados são combinadas estimativas indiretas da precipitação através dos sensores VIS/IR (visível/infravermelho) e também microondas dos satélites geoestacionários calibrados com dados de mais de 6.000 estações (Gruber e Levizzani, 2008).

O CMAP (CPC Merged Analysis of Precipitation) produz análises mensais de dados de precipitação global, onde dados de pluviômetros são combinados com as estimativas de precipitação baseadas em algoritmos e estimativas de satélites com dados de IR e PMW (microondas passivas) para a resolução de $2,5^{\circ}$ x 2,5 (lat/lon), (Xie e Arkin, 1997). A série abrange o período desde 1979. Estes dados são comparáveis com GPCP, porém não devem ser confundidos. Yin et al. (2004) realizaram uma extensa comparação entre o CMAP e o GPCP, observando que na grande escala os campos de precipitação são semelhantes, porém existem diferenças significativas em escala regional, tal como uma tendência artificial na região tropical para a série de dados do CMAP, proveniente de amostragem de dados diferentes e também o método como é feita a fusão dos dados para cada um dos produtos.

O satélite TRMM (Tropical Rainfall Measuring Mission) foi lançado em 1997 com o objetivo de monitorar e estudar a precipitação tropical e em regiões subtropicais, além de verificar como a mesma influência o clima global. Realizando varridas que se estendem desde $38^{\circ} \mathrm{N}$ a $38^{\circ} \mathrm{S}$ (Kummerow et al.,1998, 2000). Foi utilizado o produto $3 \mathrm{~B} 43$ que consiste em uma média mensal da precipitação global numa grade de $1^{\circ} \times 1^{\circ}$ (lat/lon) desde 1998. No trabalho realizado por $\mathrm{Li}$ e $\mathrm{Fu}$ (2005) foram comparados os valores médios mensais de precipitação para a região tropical com os dados provenientes das estimativas de radar do TRMM e GPCP para o período de 1998 a 2002. Onde o trabalho mostrou que as duas fontes são consistentes com relação ao padrão da distribuição da precipitação, com algumas diferenças significativas em regiões de precipitação intensa sobre os oceanos tropicais.

\subsection{Metodologia de Comparação Geral}

Os cinco bancos de dados foram trabalhados no NCL (NCAR Command Language), que é uma linguagem projetada especificamente para o processamento e visualização de dados científicos. Todos os arquivos de dados utilizados que não se encontravam em formato netCDF (Network Common Data Format) foram convertidos para este formato, antes de serem trabalhados no NCL (Tabela 1).

A primeira comparação realizada foi em termos de média de todo o período dos produtos, através de mapas com os valores plotados para o continente sul americano. Depois se realizaram 
Tabela 1 - Informações gerais dos produtos de precipitação utilizados.

\begin{tabular}{ccc}
\hline Produtos & Resolução & Formato original \\
\hline CPTEC & $0,4^{\circ} \times 0,4^{\circ}$ & Grib \\
NCEP/NCAR & $\sim 1,9^{\circ} \times 1,9^{\circ}$ & netCDF \\
GPCP & $2,5^{\circ} \times 2,5^{\circ}$ & netCDF \\
CMAP & $2,5^{\circ} \times 2,5^{\circ}$ & netCDF \\
TRMM & $1,0^{\circ} \times 1,0^{\circ}$ & hdf \\
\hline
\end{tabular}

análises espaciais mais detalhadas: variação meridional, por tipos de vegetação e por bacias hidrográficas.

\subsubsection{Análise da Variação Meridional}

Para realizar esta análise da precipitação, todos os bancos de dados foram submetidos a máscaras, em que os valores de precipitação foram plotados somente no continente e, em seguida, separados por bandas de latitude desde os $10^{\circ} \mathrm{N}$ até $50^{\circ} \mathrm{S}$, variando de $5^{\circ}$ em $5^{\circ}$, resultando em um total de 12 bandas para o continente. Com as bandas prontas calculou-se a média para cada uma das cinco séries de dados de precipitação avaliadas neste estudo.

\subsubsection{Análise por Tipo de Vegetação}

A máscara utilizada para diferenciar os tipos de vegetação que predominam na América do Sul foi criada pelo Center for Sustainability and the Global Environment da University of Wisconsin-Madison (www.sage.wisc.edu). Ao todo são representados 15 tipos de biomas para o globo todo, na resolução de $0,5^{\circ}$ x $0,5^{\circ}$ (lat/lon) (Ramankutty e Foley, 1999).

A partir da máscara original foram criadas outras máscaras, porém com resoluções diferentes $\left(1,0^{\circ} \times 1,0^{\circ}\right.$ e $2,5^{\circ}$ x $2,5^{\circ}$ ). Para fazer essa modificação de resolução foi adotado o critério de maior concentração de bioma na célula original $\left(0,5^{\circ} \times 0,5^{\circ}\right)$. Por exemplo, para converter de $0,5^{\circ}$ para $2,5^{\circ}$ criou-se uma matriz de 5 por 5 , e tomou-se a condição de que o bioma que predominasse em mais de 50\% representaria o valor da nova célula $\left(2,5^{\circ} \times 2,5^{\circ}\right)$. Procedimento semelhante foi realizado para criar a máscara de $1,0^{\circ} \times 1,0^{\circ}$. Em seguida, as máscaras (Figura 1) foram aplicadas aos dados, possibilitando calcular a média mensal de cada bioma. É importante ressaltar que ao fazer a conversão de resolução das máscaras, perde-se um pouco da qualidade da mesma, já que pequenas áreas que na máscara original são de um bioma, ao serem convertidas podem ser classificadas como outro tipo de vegetação.

\subsubsection{Análise por Bacias Hidrográficas}

Da mesma forma que foi feita a análise para os tipos de vegetação, foram realizadas análises com a máscara das bacias hidrográficas da América do Sul, obtidas do projeto R-Hydronet V1.0 (http://www.r-hydronet.sr.unh.edu). Os dados possuem formato ASCII GRID, que é um formato de arquivo texto, onde eles se encontram no sistema de coordenadas geográficas (lat/ lon), cada valor representando um código para a identificação da bacia, para cada pixel de $0,5^{\circ} \times 0,5^{\circ}$ de resolução. Também foi necessário criar máscaras novas, compatíveis com as resoluções de $1,0^{\circ} \times 1,0^{\circ}$ e $2,5^{\circ} \times 2,5^{\circ}$. O procedimento adotado foi o mesmo aplicado ao tratamento tipo de vegetação. A Figura 2 ilustra as principais bacias hidrográficas da América do Sul.

\section{RESULTADOS E DISCUSSÃO}

\subsection{Comparação dos Campos Médios}

Na Figura 3 são apresentados os campos de intensidade média de precipitação anual para o período 2000 a 2004, gerados a partir dos cinco produtos analisados neste estudo. De modo geral, observa-se que os campos de precipitação média anual dos diferentes produtos tendem a apresentar comportamento relativamente diferente entre si. Em especial, o campo de reanálise do CPTEC (Figura 3a) destaca-se por superestimar, em relação aos demais, a intensidade média da precipitação no interior do nordeste brasileiro e subestimar nas demais regiões do continente, conferindo-lhes valores extremamente baixos (abaixo de $2 \mathrm{~mm} /$ dia), o que na maioria dos lugares não 
corresponde à realidade, já que valores médios da intensidade de precipitação dessa magnitude são característicos de regiões semi-áridas. Nota-se o mesmo problema da superestimativa da intensidade média de precipitação para o nordeste brasileiro no campo da reanálise do NCEP/NCAR (Figura 3b).

Dois regimes de precipitação bem característicos no hemisfério sul são os dos oceanos Atlântico e Pacífico, onde na costa do oceano Pacífico (entre $5^{\circ} \mathrm{S}$ e $35^{\circ} \mathrm{S}$ de latitude) os valores apresentados nos campos são baixos e no Atlântico Sul são mais elevados, devido à maior temperatura oceânica do Atlântico Sul. Esta diferença pluviométrica entre os oceanos sul-americanos não é detectada na reanálise do CPTEC, mas nos demais ela é representada.

Outra característica da intensidade média da precipitação no continente é a representação dos valores altos sobre a região amazônica, principalmente na parte que se encontra sobre a Venezuela e Colômbia. Esta característica é apresentada pelos cinco produtos, porém o CMAP e GPCP (Figuras 3d e 3e) mostram valores um pouco mais baixos (entre 6 e $8 \mathrm{~mm} / \mathrm{dia}$ ), enquanto os demais apresentam valores que superam $10 \mathrm{~mm} / \mathrm{dia}$.
Para os produtos que combinam dados de estações pluviométricas com dados de satélite para gerar séries históricas de precipitação (Figuras 3c, 3d e 3e), observa-se uma forte concordância entre os produtos de CMAP e GPCP. Ambos se mostraram com boa capacidade em representar o sistema sinótico Zona de Convergência do Atlântico Sul (ZCAS), que é a faixa de valores mais elevados da intensidade média de precipitação que cobre o noroeste do continente até o sudeste/sul do Brasil, estendendo-se até o oceano. No campo gerado pelo produto do TRMM (Figura 3c) também é possível observar a presença da ZCAS, porém na região sul do Brasil e Uruguai ela apresenta uma intensidade média anual da precipitação superestimada (entre 8 a $10 \mathrm{~mm} /$ dia). Uma das possíveis causas do baixo desempenho mostrado pelas estimativas do TRMM nesta região, pode estar associada a metodologia utilizada para gerar o algoritmo 3B43, que utiliza somente informações da região espectral IR. É conhecido que a região extra-tropical e frequentemente afetada por fenômenos atmosféricos como ZCAS, Sistemas Convectivos de Mesoescala (SCM) e frentes frias, que produzem grande convecção, o que gera também

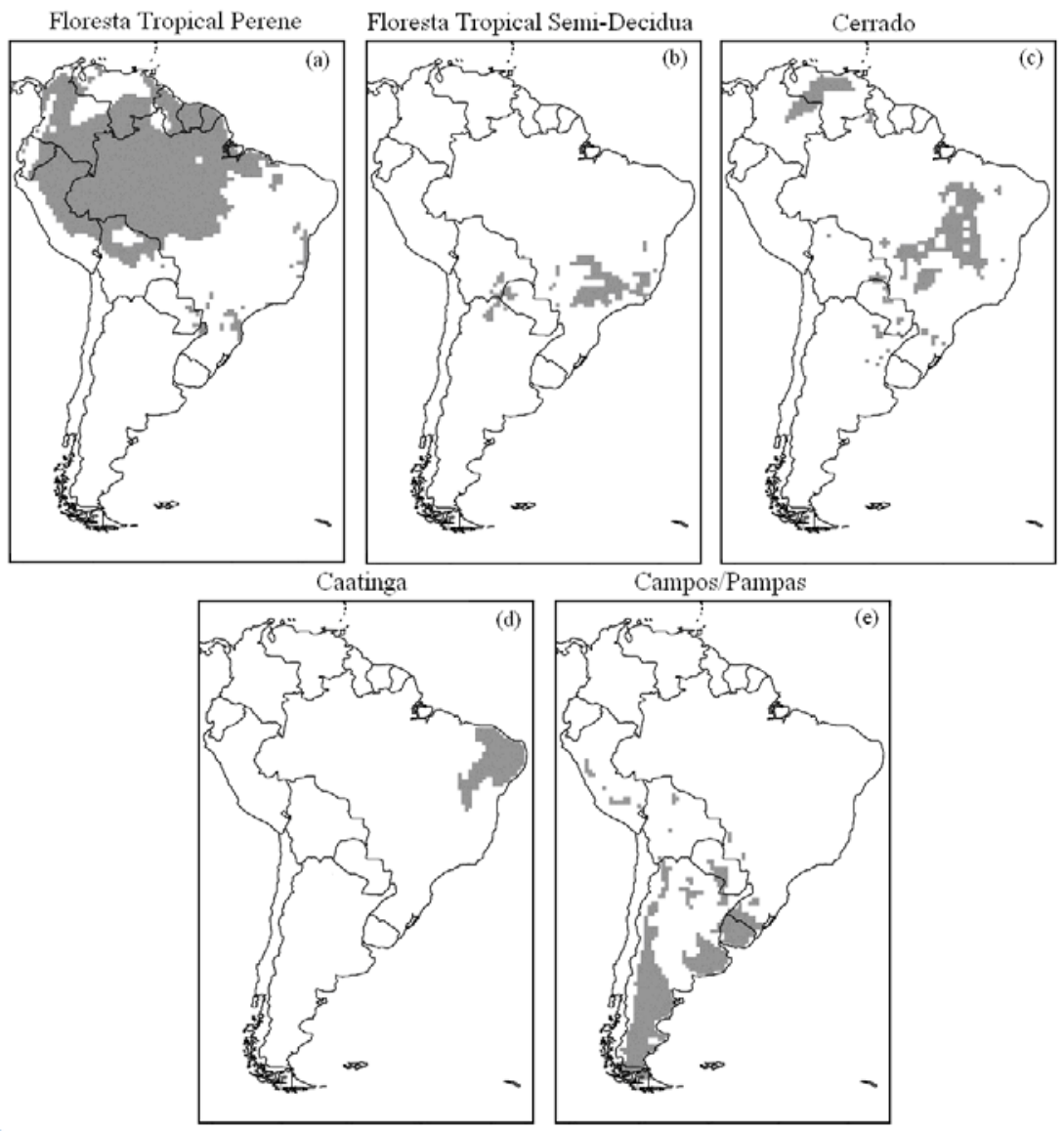

Figura 1 - Máscaras com os tipos de vegetação adotados, (a) Floresta Tropical Perene, (b) Floresta Tropical Semi-Decídua, (c) Cerrado, (d) Caatinga e (e) Campos/Pampas. 
a formação de nuvens do tipo cirrus (que são compostas basicamente por gelo), e este fenômeno pode estar relacionado à superestimativa que o produto de precipitação do TRMM apresentou nesta região.

Um trabalho que utilizou uma metodologia semelhante à empregada neste item foi o realizado por Costa e Foley (1998), que compararam diversos bancos de dados para a bacia Amazônica, onde os produtos analisados (seis ao todo) mostram que para os totais acumulados de vários anos sobre a região apresentam certa concordância, mas quando analisados ano a ano, nota-se diferenças expressivas entre as séries de dados. No presente caso as médias anuais de precipitação para a região amazônica apresentaram os valores mais elevados do continente, o que condiz com a realidade, exceto pelo fato da variação do posicionamento dos valores máximos dentro da região.

\subsection{Análise da Variação Meridional}

Analisando o gráfico das médias para as bandas de latitude (Figura 4), observa-se que dos cinco produtos considerados para a análise da intensidade media da precipitação, apenas o CPTEC encontra-se fora da faixa de valores médios para praticamente todas as bandas consideradas e o NCEP/ NCAR e TRMM encontram-se fora da faixa média apenas em determinadas bandas. Para o NCEP/NCAR, essa discordância é verificada na faixa entre $10^{\circ} \mathrm{N}$ até $5^{\circ} \mathrm{S}$, apresentando valores, aproximadamente, entre 7 a $9 \mathrm{~mm} / \mathrm{dia}$, sendo que os demais produtos para essa mesma região, têm valores entre 4 a 7 $\mathrm{mm} /$ dia. Esta diferença apresentada pelas reanálises do NCEP/ NCAR pode ser proveniente da parametrização utilizada pelos modelos numéricos que geram estes produtos, assim como também a quantidade de dados que o NCEP/NCAR utiliza para realizar a assimilação dos dados de entrada em seus modelos. Sabe-se que esta faixa de latitude é uma das que apresenta maior complexidade de representação para a modelagem numérica (sejam globais ou regionais), pois se trata de uma zona altamente convectiva e com baixa densidade de medição de dados meteorológicos. Já o TRMM apresenta valores superiores aos demais produtos para as bandas compreendidas na região entre $20^{\circ} \mathrm{S}$ a $40^{\circ} \mathrm{S}$. Estas superestimativas são ocasionadas

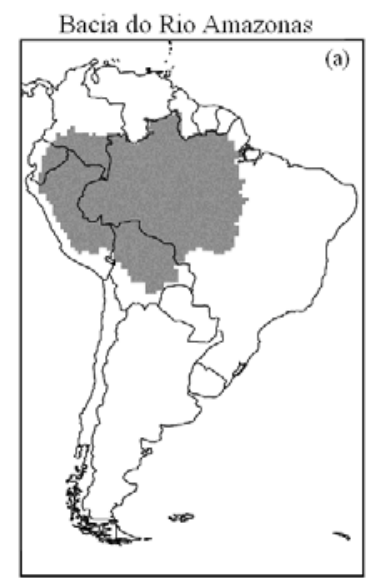

Bacia do Rio Săo Francisco

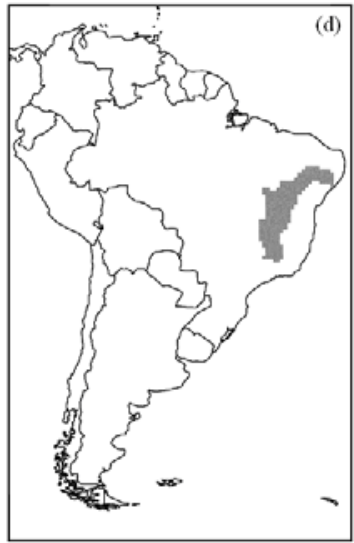

Bacia do Rio Orinoco

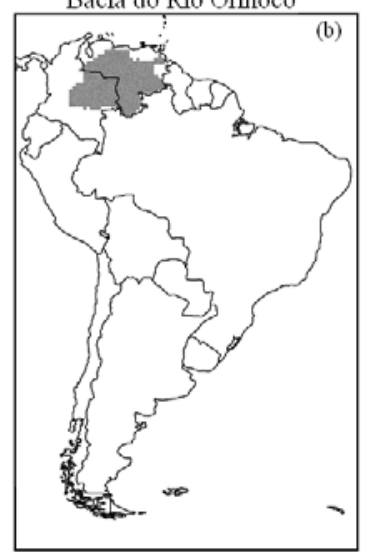

Bacia do Rio Paraná/Prata

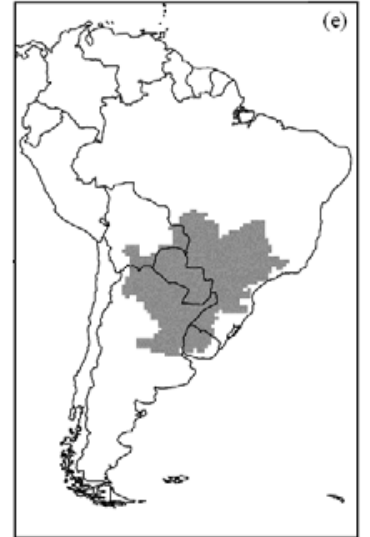

Bacia do Rio Tocantins/Araguaia

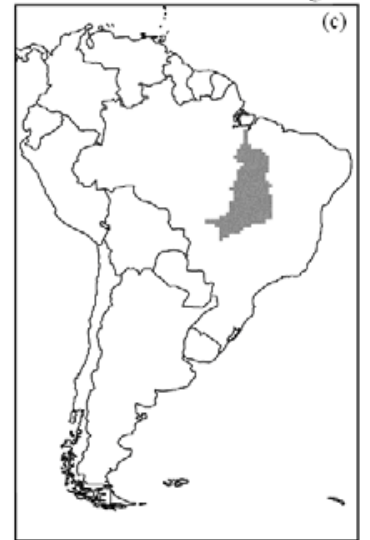

Bacia dos Rios da Patagônia

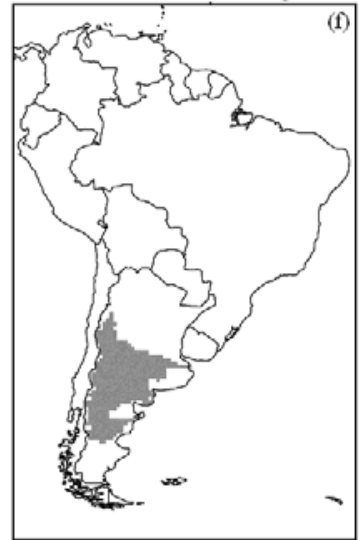

Figura 2 - Máscaras das principais bacias hidrográficas da América do Sul, (a) Bacia do Rio Amazonas, (b) Bacia do Rio Orinoco, (c) Bacia do Rio Tocantins/Araguaia, (d) Bacia do Rio São Francisco, (e) Bacia do Rio Paraná/Prata e (f) Bacia dos Rios da Patagônia. 
por dois fatores: 1) como foi mencionado anteriormente, o algoritmo 3B43 do satélite TRMM possui limitações para avaliar a precipitação em latitudes extratropicais em função dos fenômenos atmosféricos frequentemente gerados nesta faixa de latitudes; 2) falhas na cobertura sobre os Andes, o que ocasionou a obtenção de médias não representativas.

Fazendo uma análise no conjunto de dados apresentado na Figura 4, confirma-se a variação meridional esperada para a intensidade média da precipitação neste continente, que é de valores mais altos de precipitação nos trópicos e decréscimo conforme a latitude aumenta. O produto de reanálise do CPTEC consegue acompanhar o padrão meridional, mas sempre com os valores subestimados.

\subsection{Análise por Tipo de Vegetação}

A Figura 5 apresenta os valores de intensidade média mensal para cada tipo de vegetação. A análise por tipos de vegetação permite verificar se o hidroclima representado é compatível com o tipo de vegetação. Além disso, essa análise é útil para avaliação dos bancos de dados de precipitação para uso por modelos de dinâmica de vegetação.

Para a floresta tropical perene (Figura 5a) observa-se que dois produtos possuem médias mensais bem próximas para todos os meses (CMAP e GPCP). O produto de reanálise do NCEP/ NCAR mostra valores mais elevados de junho a dezembro em relação aos demais produtos. Já o produto TRMM tem valores médios intermediários entre os anteriores para os meses de agosto a dezembro. Para o produto de reanálise do CPTEC, a média da floresta tropical perene é subestimada para o ano todo, não ultrapassando a $4 \mathrm{~mm} /$ dia na estação chuvosa. Em todos os casos exceto CPTEC, o padrão de chuva é consistente com a vegetação da região, i.e., todo o ano é chuvoso, com ligeira sazonalidade e máximo anual no mês de março (Gonzales et al., 2007).

A floresta tropical semi-decídua (Figura $5 b$ ) tem seu regime de precipitação na estação seca (maio a julho) bem
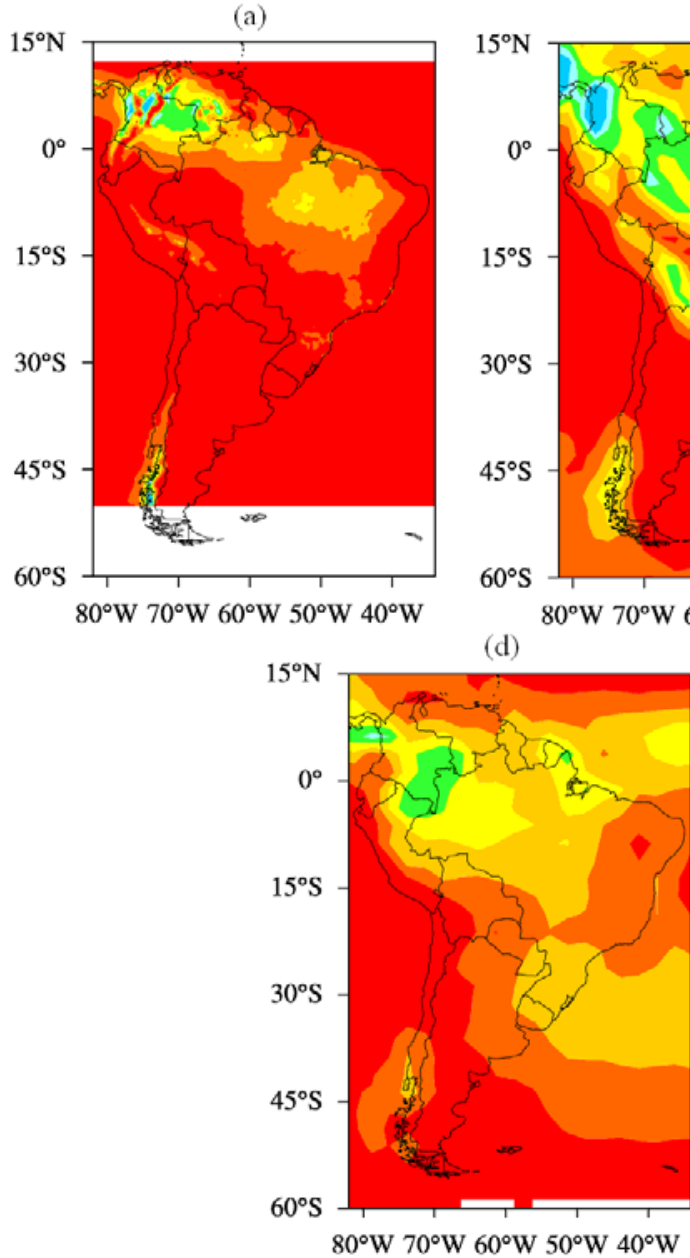

(d) (b)
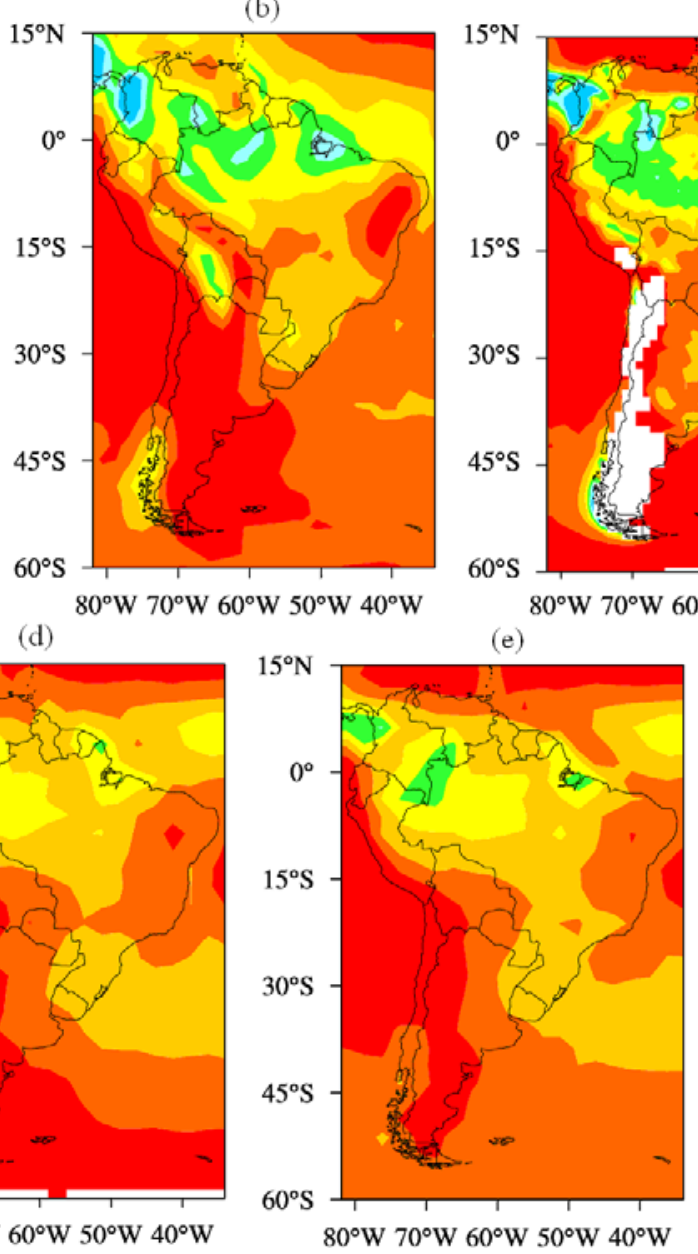

(c)

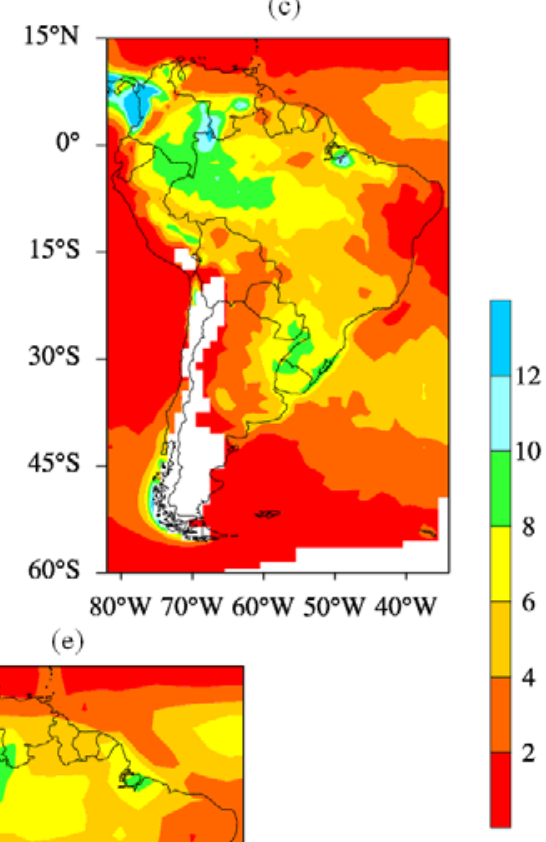

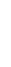


representado por quatro produtos (NCEP/NCAR, CMAP, GPCP e TRMM). Para a estação chuvosa nota-se que o produto TRMM encontra-se com seus valores médios um pouco maiores em relação aos demais. Novamente a reanálise do CPTEC mostrou-se com valores de intensidade média da precipitação mais baixa para todos os meses do ano em relação aos demais produtos. Em todos os casos exceto CPTEC, o padrão de chuva é consistente com a vegetação da região, i.e., um período chuvoso e um período seco.

No bioma caatinga, todos os produtos apresentaram valores médios próximos entre si, principalmente de agosto a dezembro (Figura 5c), com ligeira subestimativa do CPTEC em janeiro e fevereiro. Aqui, todos os padrões de chuva representados são consistentes com a vegetação xerofítica da região, i.e., um período chuvoso curto e um período seco longo.

Outra observação relevante é verificada para a curva do CPTEC no cerrado (Figura 5d), onde seus valores são subestimados apenas na estação chuvosa. O TRMM superestima a intensidade média da precipitação para os meses de agosto a dezembro, os outros três produtos (NCEP/NCAR, CMAP e GPCP) mostram resultados com valores médios bem próximos. Também aqui, em todos os casos exceto CPTEC, o padrão de chuva é consistente com a vegetação da região, com um período chuvoso e outro seco.

No caso do bioma pampas (Figura 5e), as superestimativas apresentadas pelo produto TRMM, durante o ano todo, provavelmente devem estar relacionados à freqüência de formação de SCM nas regiões sul e sudeste do Brasil, norte da Argentina, Uruguai e Paraguai, principalmente para a estação da primavera. Além disso, a falha apresentada por este produto na região dos Andes, pode contribuir para que a média permaneça alta para todos os meses do ano em relação aos outros produtos. Os pampas são caracterizados por apresentarem uma baixa sazonalidade, tendo os seus valores encontrados basicamente na faixa de 1 a $3,5 \mathrm{~mm} /$ dia. Porém, os dados da reanálise do CPTEC colocam as médias bem mais baixas em relação aos outros produtos (menos de $1 \mathrm{~mm} / \mathrm{dia}$ ). Mais uma vez, os padrões de chuva são consistentes com o tipo de vegetação para todos os casos exceto o CPTEC.

Fazendo uma análise geral da Figura 6 pode-se observar a existência de uma forte similaridade no padrão sazonal dos produtos em todos os biomas representados, apesar do produto de reanálise do CPTEC apresentar valores de intensidade médios mais baixos para a maioria dos meses em todos os produtos, com exceção do bioma caatinga.

\subsection{Análise para as Principais Bacias Hidrográficas}

A Figura 6 apresenta os valores de intensidade média mensal para cada bacia considerada neste estudo. Para a bacia do rio Amazonas (Figura 6a) pode ser visualizada uma discordância entre os valores médios mensais do CPTEC e os demais produtos. Nesta bacia, onde está o maior rio do mundo em volume de água, fica evidente a deficiência deste produto, onde o pico de intensidade média da precipitação não ultrapassou 4

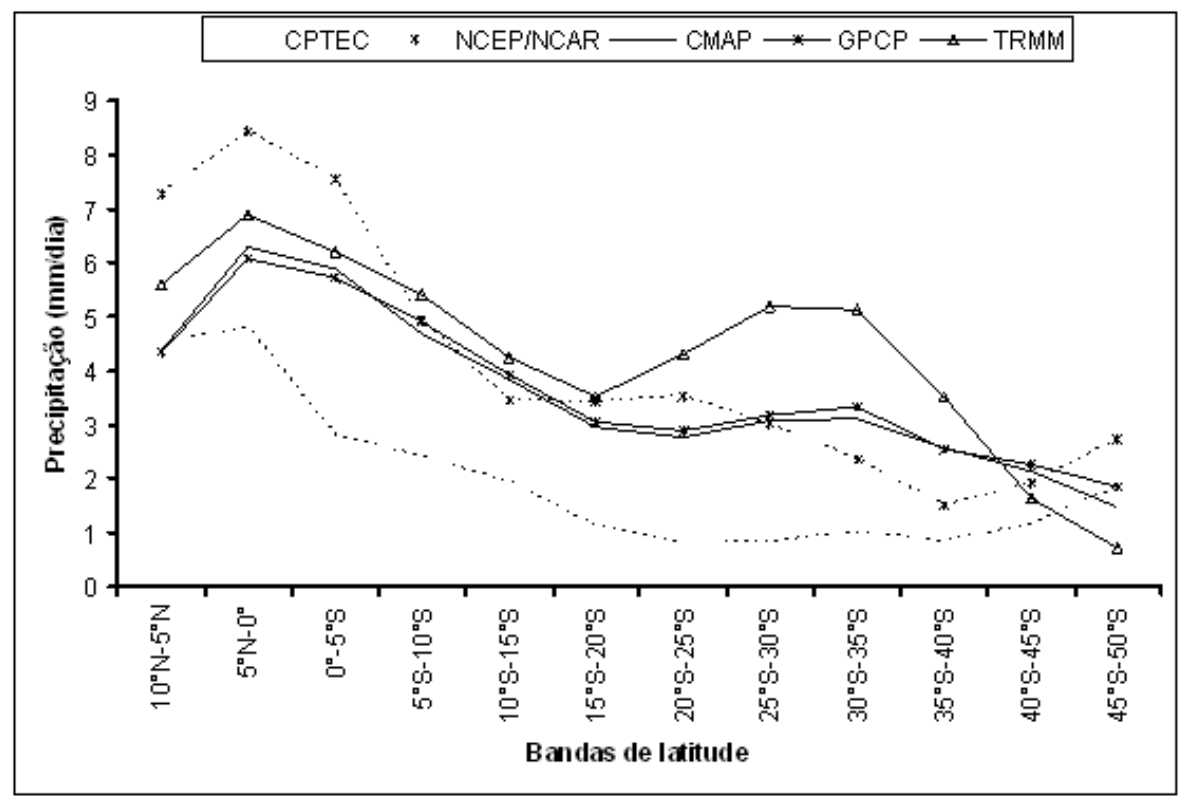

Figura 4 - Variação meridional da intensidade média da precipitação para a América do Sul. 
$\mathrm{mm} /$ dia. Outro fato interessante evidenciado nesta figura são os valores representados pela curva do produto TRMM para os meses de setembro a dezembro, onde os valores são mais elevados em relação aos demais produtos, o que condiz com a curva apresentada por este mesmo produto para a análise feita na Figura 5a.

A bacia do rio Orinoco se localiza ao norte da região amazônica (hemisférico norte) e possui regime de precipitação fora de fase em relação ao das demais bacias, devido principalmente ao deslocamento da Zona de Convergência Inter Tropical (ZCIT) do hemisfério norte para o sul (Figura 6b). As curvas dos produtos adquiridos a partir da composição de observações de superfície com sensoriamento remoto (TRMM, CMAP e GPCP), mostram uma alta concordância entre si. Os bancos de dados construídos através de reanálise para esta bacia possuem valores abaixo da média dos demais produtos na estação chuvosa, com exceção dos meses de janeiro a abril, quando a reanálise do NCEP/NCAR apresenta valores médios mais elevados em relação aos demais produtos.

Os gráficos das bacias dos rios Tocantins/Araguaia (Figura 6c) e do rio São Francisco (Figura 6d) mostram que todos os produtos possuem uma representação da estação chuvosa e seca bem definida, embora a reanálise do CPTEC seja mais seca que os demais de novembro a março na bacia do Tocantins/Araguaia. A estação seca começa a apresentar valores mais baixos no mês de maio e se estendendo até setembro. Na Figura 6c observa-se também que as curvas dos produtos de reanálise têm os valores mais elevados, principalmente na estação seca, em relação às demais curvas, porém todas as curvas apresentam o mesmo perfil ao longo do ano. (a) Floresta Tropical Perene

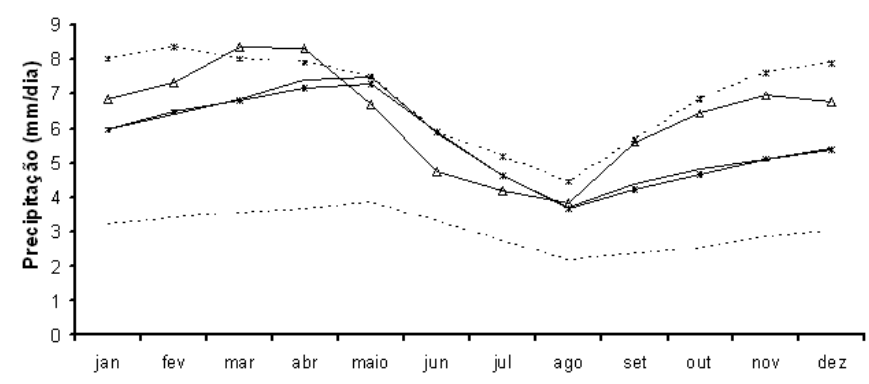

(c) Caatinga

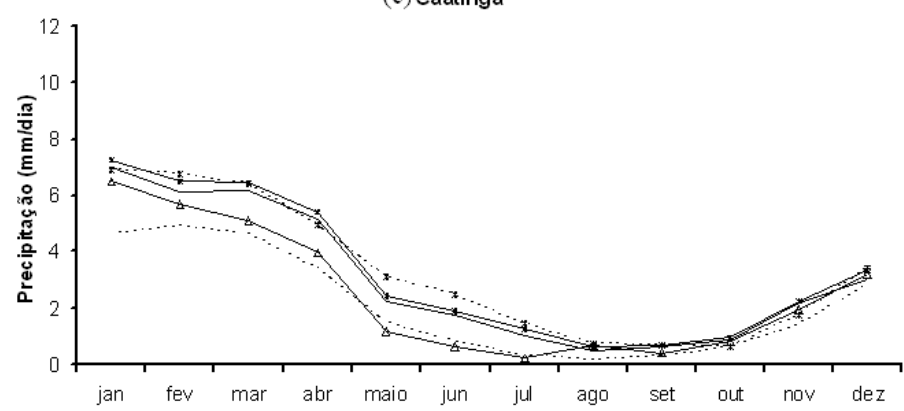

(e)Pampas/Campos

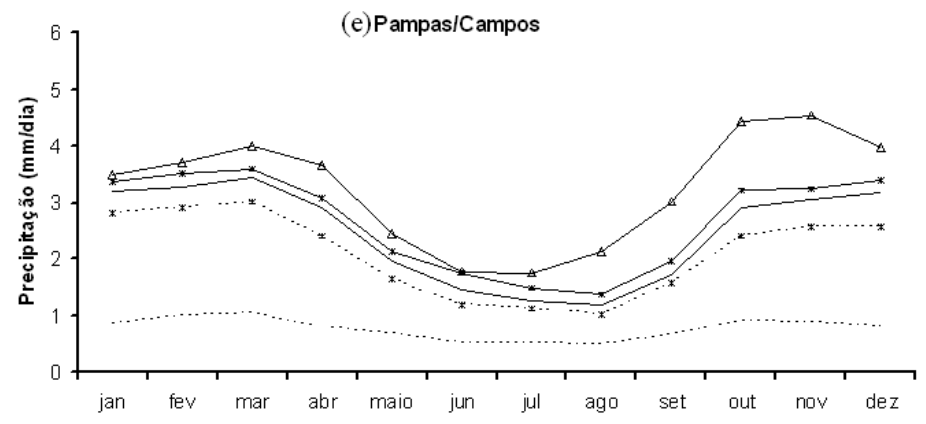

(b) Floresta Tropical Semi-Decidua

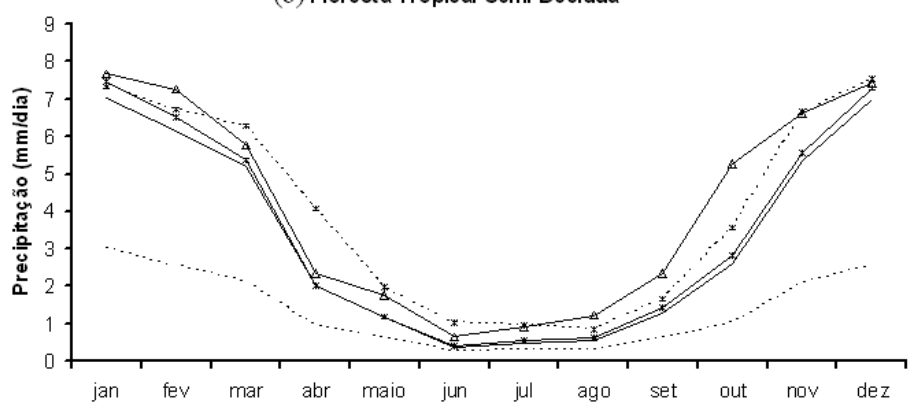

(d) Cerrado

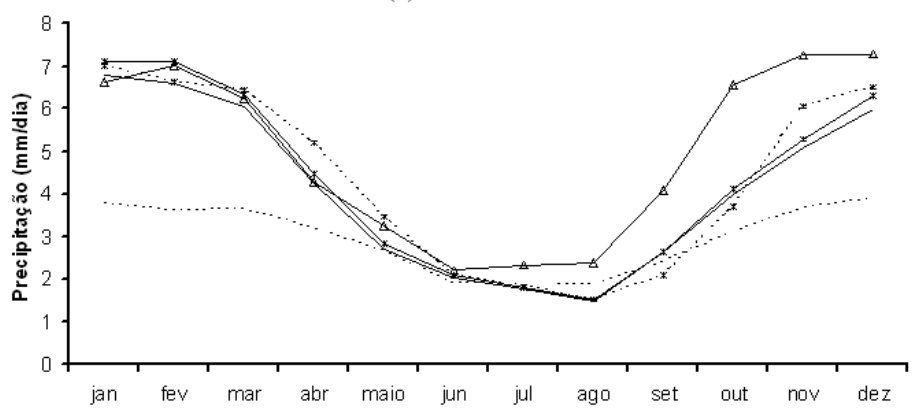

Figura 5 - Médias mensais da intensidade média da precipitação para os principais tipos de vegetação da América do Sul, (a) Floresta Tropical Perene, (b) Floresta Tropical Semi-Decídua, (c) Caatinga, (d) Cerrado e (e) Pampas/Campos. 
Na bacia do rio São Francisco (Figura 6d) observa-se que todos os produtos utilizados para analisar a sazonalidade da intensidade média da precipitação possuem uma forte concordância entre os dados provenientes da composição de dados observados com satélite (CMAP, GPCP e TRMM). Para os dados de reanálise nota-se que no período de janeiro a maio ocorrem valores médios mais altos que os demais produtos.

A Figura 6e mostra a deficiência do produto de reanálise do CPTEC para a bacia do rio Paraná/Prata, pois os valores médios mensais encontram-se totalmente subestimados em relação aos demais produtos. Outro que difere dos demais é o TRMM, por colocar na maioria dos meses (exceto junho e julho) valores mais altos para as médias da intensidade de precipitação mensal. Segundo Velasco e Fritsch (1987), durante a primavera e início do outono ocorre maior formação de SCM na região onde esta localizada a bacia do Rio Paraná/Prata, o que está relacionado com as discussões feitas anteriormente sobre o produto do TRMM na presença destes fenômenos atmosféricos em latitudes extratropicais.

Outro resultado curioso foi o encontrado para as bacias dos principais rios da Patagônia (Figura 6f), onde foi detectado o maior índice de discordância entre os padrões das curvas dos produtos, embora a variação sazonal seja razoavelmente pequena para todos os produtos. Como exceção, o produto de (a) Bacia do Rio Amazonas

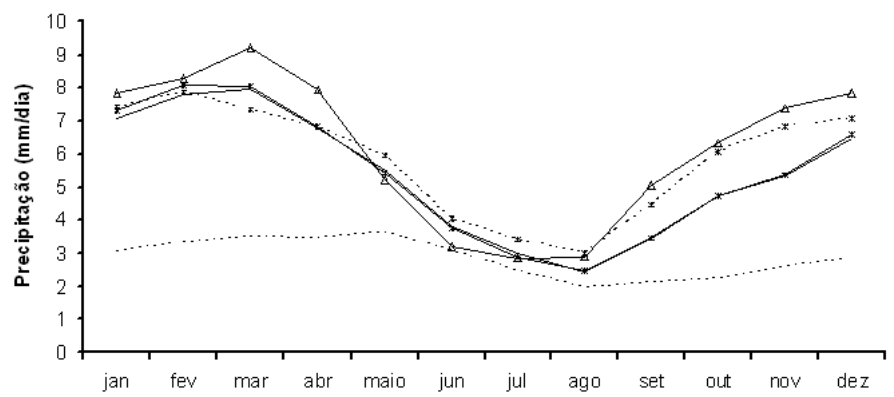

(c) Bacia do Rio Toc antins/Araguaia

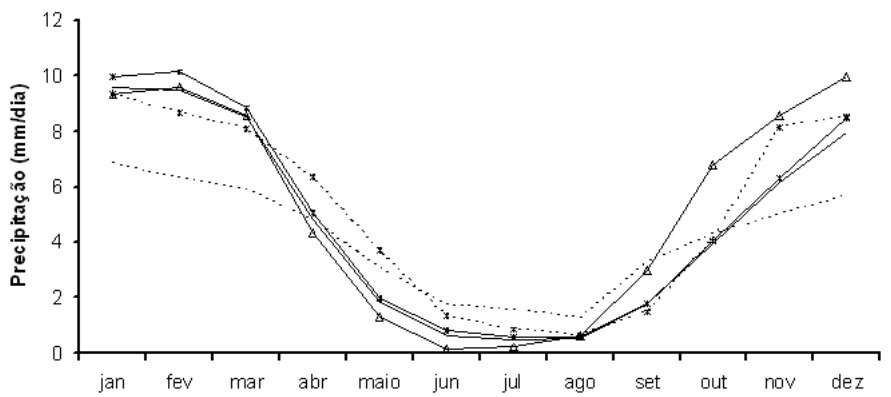

(e) Bacia do Rio Paraná - Prata

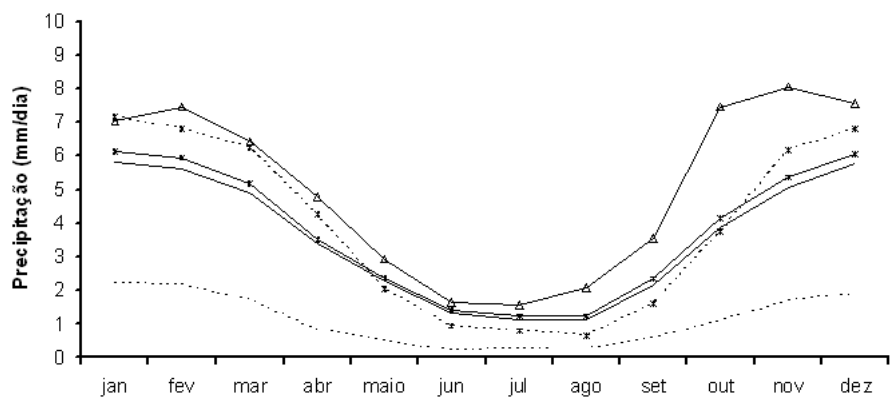

(b) Bacia do Rio Orinoco

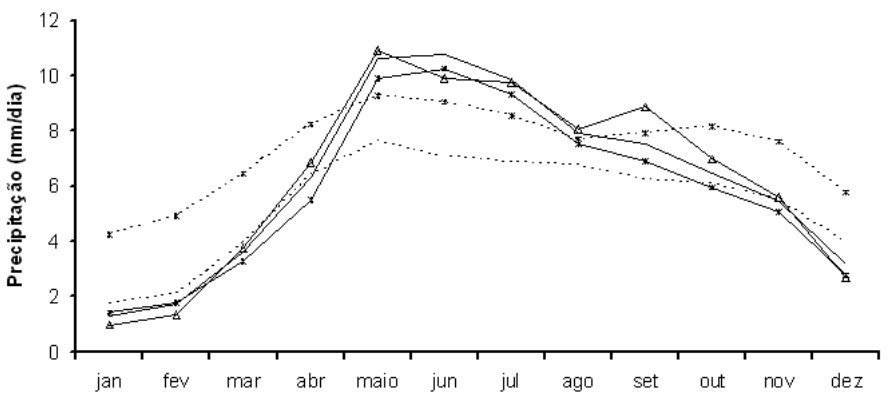

(d) Bacia do Rio São Francisco

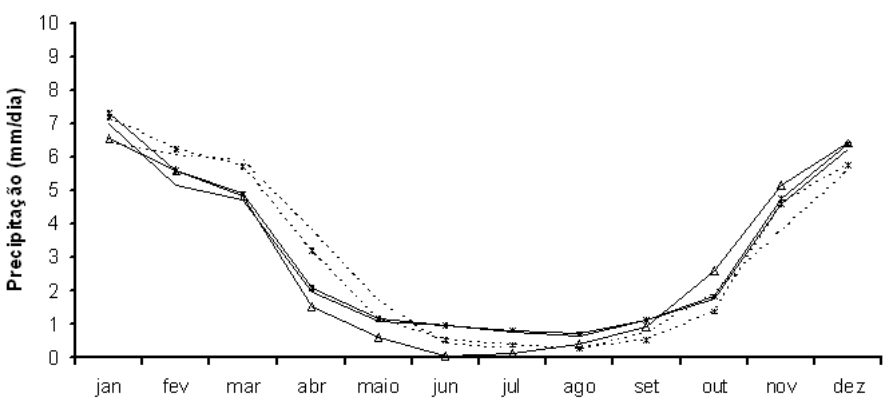

(f) Bacia dos Rios da Patagônia

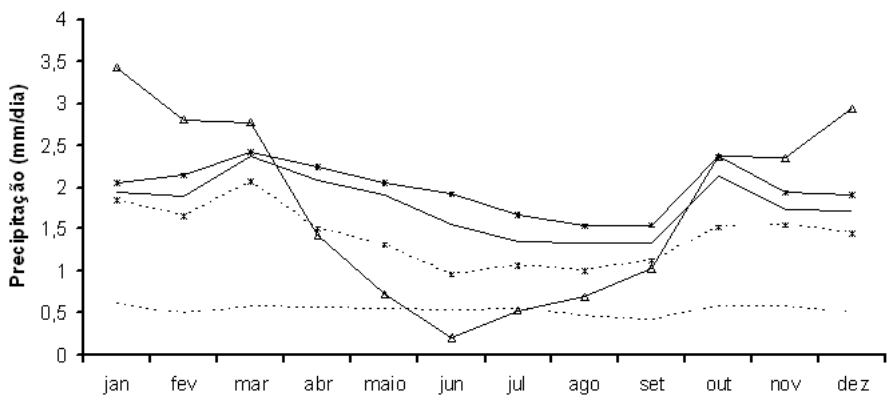

$\cdots \cdots$ CPTEC $\cdots \cdots$ NCEPNCAR - CMAP — - GPCP $₫$ TRMM

Figura 6 - Médias mensais para as principais bacias hidrográficas da América do Sul, (a) Bacia do Rio Amazonas, (b) Bacia do Rio Orinoco, (c) Bacia do Rio Tocantins/Araguaia, (d) Bacia do Rio São Francisco, (e) Bacia do Rio Paraná/Prata e (f) Bacia dos Rios da Patagônia. 
satélite TRMM, que mostra uma forte sazonalidade, onde a intensidade média da precipitação varia de menos de $0,5 \mathrm{~mm} /$ dia (junho) a quase $3,5 \mathrm{~mm} /$ dia (janeiro), o que deixa claro mais uma vez as limitações deste produto para regiões de latitudes mais altas. Os demais produtos variam entre 0,5 a $2,5 \mathrm{~mm} /$ dia, porém esta diferença é mais entre um produto e outro. Observa-se que as curvas do CMAP e GPCP são paralelas entre si para todos os meses, o que mostra uma boa concordância destes produtos para as latitudes médias.

Na Figura 6 verifica-se que os padrões de sazonalidade são seguidos por todos os produtos para todas as bacias com exceção da bacia dos Rios da Patagônia (Figura 6f), onde há discordâncias significantes entre os produtos, e com exceção para o produto de reanálise do CPTEC para a bacia do rio Amazonas (Figura 6a).

\section{CONCLUSÕES E RECOMENDAÇÕES}

Neste trabalho foram comparados cinco produtos de precipitação para a América do Sul. É importante esclarecer que não foi adotado nenhum produto como referência, pois um dos propósitos deste estudo é identificar um ou mais produtos que possa vir a ser tratado como uma referência, para os diferentes tipos de vegetação, bacias hidrográficas e variação meridional.

Os usuários de produtos decorrentes do satélite TRMM precisam ter bastante cautela ao utilizarem estes dados para latitudes extratropicais, pois para todas as análises realizadas neste estudo onde as regiões se encontravam fora dos trópicos, os valores apresentados pelo TRMM foram mais altos em relação à maioria dos produtos e, em alguns casos, a sazonalidade estava comprometida. No caso do produto de reanálise do CPTEC ficou clara a sua deficiência em estimar a precipitação, que foi subestimada em praticamente todas as análises. Esta deficiência pode estar relacionada com a parametrização de nuvens do modelo Eta.

$\mathrm{Na}$ análise realizada para a variação meridional do continente nota-se que dois produtos apresentaram médias bem próximas (CMAP e GPCP), enquanto os outros três produtos mostraram-se com seus valores médios deslocados, sendo que a reanálise NCEP/NCAR superestima a intensidade média da precipitação para a faixa de latitude de $10^{\circ} \mathrm{N}$ a $5^{\circ} \mathrm{S}$, o TRMM superestima os valores para a faixa de latitude de $20^{\circ} \mathrm{S}$ a $40^{\circ} \mathrm{S}$, e a reanálise do CPTEC subestimou as médias para todas as bandas de latitude.

Para a análise dos tipos de vegetação e bacias hidrográficas, observa-se que de modo geral os produtos de precipitação avaliados conseguiram representar de forma expressiva a sazonalidade existente para estas regiões. Novamente detectouse que os produtos derivados da combinação de dados de satélite, principalmente o CMAP e GPCP, apresentam uma boa concordância entre as suas médias.

Dada a importância deste estudo, no que diz respeito à escolha de um produto de precipitação para estudos de modelagem hidrológica e ecológica, algumas recomendações específicas para os tipos de vegetação e bacias hidrográficas aqui analisadas se fazem necessárias:

- Tipos de Vegetação: Notou-se que para todos os biomas os produtos que sempre apresentam médias próximas em todos os meses são CMAP e GPCP. Porém em alguns casos específicos verifica-se que outros estão dentro da média da maioria, como para o bioma caatinga onde o produto de reanálise do CPTEC também apresenta resultados coerentes.

- Bacias Hidrográficas: Para a bacia do rio Amazonas os produtos que apresentaram uma melhor concordância foram o CMAP e GPCP, já para a bacia do rio Orinoco foram três produtos (CMAP, GPCP e TRMM) que mostraram resultados semelhantes. Para as bacias do rio Tocantins/Araguaia e rio Paraná/Prata as médias mensais dos produtos CMAP e GPCP apresentam resultados bem próximos com maior concordância. Na bacia do rio São Francisco os produtos de sensoriamento remoto CMAP e GPCP e a reanálise do CPTEC mostraram uma forte concordância entre si. Para a bacia dos rios da Patagônia os resultados encontrados não permitem fazer recomendações para futuros usuários destes produtos, sem antes serem realizados mais estudos nesta região.

\section{REFERÊNCIAS BIBLIOGRÁFICAS}

COSTA, M. H.; J. A. FOLEY. A comparison of precipitation datasets for the Amazon basin. Geophysical Research Letters, v. 25, p. 155-158, 1998.

GONZÁLEZ, M. et al. The nature of the rainfall onset over central South America. Atmósfera, v. 20(4), p. 377-394, 2007.

GRUBER, A.; V. LEVIZZANI. Assessment of Global Precipitation Products. A project of the World Climate Research Programme Global Energy and Water Cycle Experiment (GEWEX) Radiation Panel. WCRP Report, 2008.

HUFFMAN, G. J. et al. The global precipitation climatology project (GPCP) combined precipitation dataset. Bulletin of the American Meteorological Society, v. 78, p. 5-20, 1997.

KALNAY, E. et al. The NCEP/NCAR 40-Year Reanalysis project. Bulletin of the American Meteorological Society, v. 77, p. 437-471, 1996.

KUMMEROW, C. D. et al. The status of the Tropical Rainfall Measuring Mission (TRMM) after two years in orbit. Journal of Applied Meteorology, v. 39, p. 1965-1982, 2000. 
KUMMEROW, C. et al. The tropical rainfall measuring mission (TRMM) sensor package. Journal of Atmospheric and Oceanic Technology, v. 15, p. 809-817, 1998.

LEEMANS, R.; W. P. CRAMER. The IIASA database for mean monthly values of temperature, precipitation and cloudiness on a global terrestrial grid. IIASA Working Papers, WP90-41, Laxenburg, Austria, 1990.

LEGATES, D. R.; C. J. WILLMOTT. Mean seasonal and spatial variability in gauge-corrected, global precipitation. International Journal of Climatology, v. 10, p. 111-127, 1990.

LI, R.; Y. FU. Tropical Precipitation Estimated by GPCP and TRMM PR Observations. Advances in Atmospheric Sciences, v. 22, p. 852-864, 2005.

LORENZ, E. N. The nature and theory of the general circulation of atmosphere. World Meteorological Organization, $n$. 218, Geneva, 1969.

NEW, M.; M. HULME; P. JONES. Representing twentieth century spacetime climate variability. Part 1: development of a 1961-90 mean monthly terrestrial climatology. Journal of Climate, v. 12, p. 829-856, 1999.

POHLMANN, H.; R.J. GREATBATCH. Discontinuities in the late 1960's in different atmospheric data products. Geophysical Research Letters, v.33, L22803, doi:10.1029/ 2006GL027644, 2006.
RAMANKUTTY, N.; J. A. FOLEY. Estimating historical changes in global land cover: croplands from 1700 to 1992. Global Biogeochemical Cycles, v. 13, p. 997-1027, 1999.

RAO, V. B.; I. F.A. CAVALCANTI; K. HADA. Annual variations of rainfall over Brazil and water vapor characteristics over South America. Journal of Geophysical Research, v.101, p.26539-26552, 1996.

ROADS, J. O. et al. Surface water characteristics in NCEP global spectral model and reanalysis. Journal of Geophysical Research-Atmospheres, v. 104, p. 19307-19327, 1999.

UPPALA, S. M. et al. The ERA-40 re-analysis. Quarterly Journal of the Royal Meteorological Society, v. 131, p. 2961-3012, doi:10.1256/qj.04.176, 2005.

VELASCO, I.; J. M. FRITSCH. Mesoscale convective complexes in the Americas. Journal of Geophysical Research, v. 92, p. 9591-9613, 1987.

XIE, P., P. A. ARKIN. Global precipitation: a 17-year monthly analysis based on gauge observations, satellite estimates, and numerical model outputs. Bulletin of the American Meteorological Society, v. 78, p. 2539-2558, 1997.

YIN, X.; A. GRUBER; P. A. ARKIN. Comparison of the GPCP and CMAP merged gauge-satellite monthly precipitation products for the period 1979-2001. Journal of Hydrometeorology, v. 5, p. 1207-1222, 2004. 